\title{
Direct Time-domain Channel Impulse Response Estimation for OFDM-based Systems
}

\author{
Carlos Ribeiro \\ Polytechnic Institute of Leiria \\ Leiria, Portugal \\ cribeiro@estg.ipleiria.pt
}

\author{
Atílio Gameiro \\ Instituto de Telecomunicações \\ University of Aveiro \\ Aveiro, Portugal \\ amg@det.ua.pt
}

\begin{abstract}
A method to estimate the channel impulse response using the received serial data in time-domain, without using DFT operations, is investigated. The processing required by the proposed method is substantially reduced when compared with LS-DFT channel estimation method, with no performance degradation. Furthermore, this method can incorporate the results of other published works that are DFT-based, at little or no added complexity, to improve the estimate of channel impulse response.
\end{abstract}

Keywords-OFDM, pilot-aided channel estimation.

\section{INTRODUCTION}

Orthogonal Frequency Division Multiplexing (OFDM) is the choice for a variety of wideband applications due to its robustness against frequency selective channels [1], common in mobile personal communications. OFDM systems require accurate channel estimation both for resource allocation and demodulation/decoding. Usually, to assist in the channel estimation process, some dedicated subcarriers convey deterministic symbols, commonly denominated pilots. Though decreasing the system's efficiency (since no user data is conveyed by pilot symbols), pilot-assisted channel estimation methods provide better performance than blind methods.

The typical operation at the receiver is to convert the received serial data to the frequency domain (FD) using a fast Fourier transform (FFT), calculate the estimate of the channel at the pilot positions and then perform an interpolation, using some criteria for the interpolation filter, to get the estimate of the channel's frequency response for all the subcarriers.

Published work on time domain (TD) channel estimation [2], [3] put in evidence that the estimation process can be performed directly in TD, processing the received serial signal, but due to the pilot arrangement it is more natural to move to the FD and to do the processing there. In fact, processing in the TD can be significantly simpler. It is easy to show, that the impulse response of the multiband bandpass filter that corresponds to the extraction of the pilots is also a train of equal amplitude pulses in the TD, and that the corresponding convolution operation is nothing more than a simple averaging.

In this paper, a presentation is made of a TD method that performs an equivalent Least Squares - discrete Fourier transform (LS-DFT) channel estimation [4] with considerably reduced complexity. The processing is done directly on the time received data, eliminating the majority of operations inherent to the method in [4] and other DFT-based interpolation methods.

The paper is organized as follows. Next Section presents the system model and assumptions, followed in Section III by a description of the proposed method. Simulation results and discussions are provided in Section IV. Finally, conclusions are drawn in Section V.

\section{SYSTEM DESCRIPTION}

Let us consider OFDM modulation over $N c$ subcarriers for transmission over a multipath Rayleigh fading wireless channel, with a discrete-time impulse response given by

$$
\tilde{h}[n]=\sum_{l=0}^{L-1} \alpha_{l} \delta\left[n-\tau_{l}\right]
$$

where $L$ is the number of channel paths, $\alpha_{l}$ is the complex value of path $l$ and $\tau_{l}$ is the delay of path $l$. The paths are assumed to be statistically independent, with normalized average power, $\sum_{l=0}^{L-1} \sigma_{l}^{2}=1$, where $\sigma_{l}^{2}$ is the average power of path $l$. The time dependence of the channel impulse response (CIR) is not present in the notation for simplicity.

Throughout the text, the notation $(\sim)$ is used for TD vectors and elements and its absence denotes FD vectors and elements. The index $n$ denotes TD elements and $k$ FD elements.

Suppose that the receiver is perfectly synchronized and that the insertion of a long enough cyclic prefix in the transmitter assures that the orthogonality of the subcarriers in maintained after transmission.

The transmitter multiplexes pilots along with data symbols in FD within some symbols. The FD complex transmitted column $N c$-vector s carrying pilot and data symbols can be expressed as $\mathbf{s}=\mathbf{d}+\mathbf{p}$, whose symbols belong to a twodimensional $M$-ary PSK or QAM constellation and where d

This work is supported by Fundação para a Ciência e a Tecnologia project "FFT-XXI" (ref. POSI/EEA-CPS/58759/2004). 
and $\mathbf{p}$ are column $N c$-vectors containing the data symbols and pilots symbols, respectively. Vectors $\mathbf{d}$ and $\mathbf{p}$ contain non-zero values at disjoint subcarriers.

\section{DiRECT TD CHANNEL IMPULSE RESPONSE ESTIMATION}

For the proposed method, the set of subcarriers dedicated to transmit pilots is

$$
\wp=\{0, N f, 2 N f, \cdots, N c-N f\},
$$

where $N f$ is the number of subcarriers that separate consecutive pilots in FD. The $k$-th element of $\mathbf{p}$ is defined as

$$
p[k]=\sum_{m=0}^{N t-1} \delta[k-m N f],
$$

where

$$
N t=\frac{N c}{N f} \in \mathbb{N}
$$

Define the $N c \times N c$ DFT and Inverse DFT (IDFT) matrices $\mathbf{Q}$ and $\mathbf{Q}^{-1}$, respectively, as

$$
\begin{gathered}
\mathbf{Q} \triangleq\left(e^{-j 2 \pi k n / N c}\right)_{k, n=0,0}^{N c-1, N c-1}, \\
\mathbf{Q}^{-1} \triangleq \frac{1}{N c}\left(e^{j 2 \pi k n / N c}\right)_{k, n=0,0}^{N c-1, N c-1} .
\end{gathered}
$$
as

$$
\tilde{\mathbf{s}}=\mathbf{Q}^{-1} \mathbf{s}=\mathbf{Q}^{-1}(\mathbf{d}+\mathbf{p})=\mathbf{Q}^{-1} \mathbf{d}+\mathbf{Q}^{-1} \mathbf{p}=\tilde{\mathbf{d}}+\tilde{\mathbf{p}}
$$

where $\tilde{\mathbf{d}}$ and $\tilde{\mathbf{p}}$ are TD column $N c$-vectors that collect, respectively, the components of data symbols and pilot symbols present in $\tilde{\mathbf{s}}$. The $n$-th element of $\tilde{\mathbf{s}}$ can be expressed as

$$
\tilde{s}[n]=\tilde{d}[n]+\tilde{p}[n],
$$

where $\tilde{p}[n]$ is the $n$-th element of the pilot vector $\tilde{\mathbf{p}}$,

$$
\begin{aligned}
\tilde{p}[n] & =\frac{1}{N c} \sum_{k=0}^{N c-1} \sum_{m=0}^{N t-1} \delta[k-m N f] e^{j 2 \pi \frac{k n}{N c}} \\
& =\frac{1}{N f} \sum_{m=0}^{N f-1} \delta[n-m N t]
\end{aligned}
$$

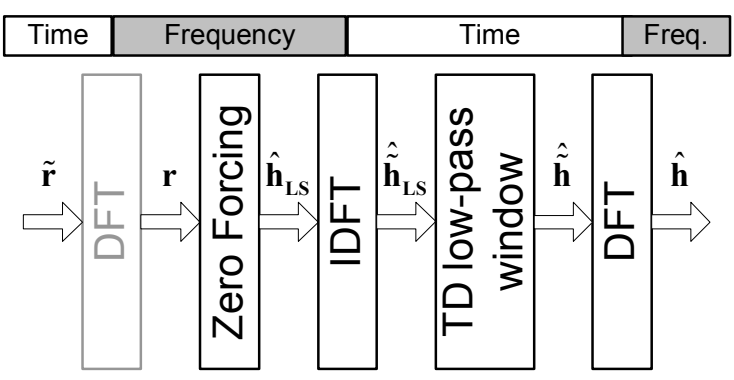

Fig. 1. OFDM pilot-aided DFT-based channel estimation.

and $\tilde{d}[n]$ is the $n$-th element of $\tilde{\mathbf{d}}$,

$$
\tilde{d}[n]=\frac{1}{N c} \sum_{\substack{k=0 \\ k \notin \wp}}^{N c-1} d[k] e^{j 2 \pi \frac{k n}{N c}},
$$

where $d[k]$ is the complex data symbol conveyed by the $k$-th subcarrier.

The transmission of $\mathbf{s}$ through an wireless channel results in the FD received signal column $N c$-vector $\mathbf{r}$, whose $k$-th element can be represented by

$$
r[k]=h[k] s[k]+w[k],
$$

where $h[k]$ is the complex value of the channel at the $k$-th subcarrier, $s[k]$ is the $k$-th element of $\mathbf{s}$ and $w[k]$ is the $k$-th element of the noise column $N c$-vector $\mathbf{w}$.

The $n$-th element of the TD received signal column $\mathrm{Nc}$ vector $\tilde{\mathbf{r}}$ can be expressed by

$$
\tilde{r}[n]=\sum_{l=-\infty}^{+\infty} \tilde{h}[l] \tilde{d}[n-l]+\frac{1}{N f} \sum_{m=0}^{N f-1} \tilde{h}[n-m N t]+\tilde{w}[n],
$$

where $\tilde{h}[n]$ is the $n$-th element of the $L$ taps CIR vector $\tilde{\mathbf{h}}$ and $\tilde{w}[n]$ is the $n$-th element of the column $N c$-vector $\tilde{\mathbf{w}}$, made-up of independent and identically distributed (iid) zero mean Additive White Gaussian Noise (AWGN) samples with variance $\sigma_{\tilde{w}}^{2}$.

The vector $\tilde{\mathbf{r}}$ is the sum of three distinct components: the data vector $\tilde{\mathbf{d}}$ and pilot vector $\tilde{\mathbf{p}}$, transmitted through the channel, and the AWGN vector $\tilde{\mathbf{w}}$. The component resulting from the transmission of pilots is made-up of $N f$ scaled replicas of the CIR, separated by $N t$ samples. The CIR replicas will not overlap if the pilot distance in frequency $N f$ fulfills the sampling theorem. 
Given the normalized channel bandwidth $\frac{\tau_{\max }}{N c \Delta t}$ [5], where $\tau_{\max }$ is the one-sided maximum delay spread and $\Delta t$ is the sampling interval, the minimum pilot distance that fulfills the sampling theorem, thus allowing for proper channel estimation, without aliasing, is

$$
N f \leq \frac{N c \Delta t}{\tau_{\max }} \Leftrightarrow N t \geq \frac{\tau_{\max }}{\Delta t}
$$

An OFDM receiver using the channel estimation method of [4], whose block diagram is depicted in Fig. 1, starts by transforming the TD vector $\tilde{\mathbf{r}}$ into the FD column $N c$-vector $\mathbf{r}$ using a DFT, to further process the received symbols. The channel estimator initiates the processing by calculating the FD LS column $N c$-vector $\hat{\mathbf{h}}_{\mathrm{LS}}$ from $\mathbf{r}$. The $k$-th element of vector $\hat{\mathbf{h}}_{\text {LS }}$ can be expressed by

$$
\begin{aligned}
\hat{h}_{L S}[k] & =\frac{r[k]}{p[k]}=(h[k]+d[k] h[k]+w[k]) \sum_{m=0}^{N t-1} \delta[k-m N f] \\
& =(h[k]+w[k]) \sum_{m=0}^{N t-1} \delta[k-m N f],
\end{aligned}
$$

recalling that pilot and data have non-zero values at disjoint subcarriers. The vector $\hat{\mathbf{h}}_{\mathrm{LS}}$ is then transformed to TD using an IDFT, resulting in the TD column $N c$-vector $\hat{\tilde{\mathbf{h}}}_{\mathrm{LS}}$. The CIR estimate column $N c$-vector $\hat{\tilde{\mathbf{h}}}$ is obtained by applying a lowpass window to $\hat{\tilde{\mathbf{h}}}_{\mathrm{LS}}$, with a cut-off frequency

$$
f_{\text {cut }-o f f}=\frac{N c}{N f}-1=N t-1,
$$

and gain $N f$. The $n$-th element of $\hat{\tilde{\mathbf{h}}}$ is

$$
\hat{\tilde{h}}[n]=\left\{\begin{array}{l}
N f \cdot \tilde{h}_{L S}[n], \quad n \in\{0,1, \cdots, N t-1\} \\
0, \quad \text { remaining }
\end{array} .\right.
$$

The channel's frequency response estimate column $\mathrm{Nc}$ vector $\hat{\mathbf{h}}$ is the result of transforming the CIR estimate back to FD,

$$
\hat{\mathbf{h}}=\mathbf{Q} \hat{\tilde{\mathbf{h}}}
$$

The channel estimation method depicted in Fig. 1 can be performed in TD up to the CIR estimate and only then be transformed to FD, to obtain the channel's frequency response $\hat{\mathbf{h}}$. In such a scenario, the $n$-th element of the vector $\hat{\tilde{\mathbf{h}}}_{\mathrm{LS}}$ can be determined from vector $\tilde{\mathbf{r}}$ (all pilots have the value " 1 "),

$$
\begin{aligned}
\hat{\tilde{h}}_{L S}[n] & =\tilde{p}[n] * \tilde{r}[n] \\
& =\frac{1}{N f} \sum_{l=-\infty}^{+\infty} \tilde{h}[l] \sum_{m=0}^{N f-1} \delta[n-m N t] * \tilde{d}[n-l] \\
& +\frac{1}{N f^{2}} \sum_{m=0}^{N f-1} \delta[n-m N t] * \sum_{l=0}^{N f-1} \tilde{h}[n-l N t] \\
& +\frac{1}{N f} \sum_{m=0}^{N f-1} \tilde{w}[n-m N t] \\
& =\frac{1}{N f} \sum_{l=-\infty}^{+\infty} \tilde{h}[l] \sum_{m=0}^{N f-1} \tilde{d}[n-m N t-l] \\
& +\frac{1}{N f} \sum_{m=0}^{N f-1} \tilde{h}[n-m N t]+\tilde{w}_{L S}[n]
\end{aligned}
$$

where

$$
\tilde{w}_{L S}[n]=\frac{1}{N f} \sum_{m=0}^{N f-1} \tilde{w}[n-m N t]
$$

is the $n$-th component of the noise column $N c$-vector $\tilde{\mathbf{w}}_{\mathrm{LS}}$, made-up of iid zero mean AWGN samples with variance $\sigma_{\tilde{w}_{L S}}^{2}=\frac{\sigma_{\tilde{w}}^{2}}{N f}$.

Considering that

$$
\begin{aligned}
& \sum_{m=0}^{N f-1} \tilde{d}[n-m N t-l]=\frac{1}{N c} \sum_{m=0}^{N f-1} \sum_{\substack{k=0 \\
k \notin \wp}}^{N c-1} d[k] e^{j 2 \pi k(n-m N t-l) / N c} \\
& =\frac{1}{N c} \sum_{\substack{k=0 \\
k \notin \wp}}^{N c-1} d[k] e^{j 2 \pi k(n-l) / N c} \sum_{m=0}^{N f-1} e^{-j 2 \pi k m N t / N c}=0
\end{aligned}
$$

equation (18) can be further simplified,

$$
\tilde{h}_{L S}[n]=\frac{1}{N f} \sum_{m=0}^{N f-1} \tilde{h}[n-m N t]+\tilde{w}_{L S}[n],
$$

and the final expression of (16) is

$$
\begin{aligned}
\tilde{\tilde{h}}[n] & =\left\{\begin{array}{l}
\sum_{m=0}^{N f-1} \tilde{h}[n-m N t]+\sum_{m=0}^{N f-1} \tilde{w}[n-m N t], n \in\{0,1, \cdots, N t-1\} \\
0, \quad \text { remaining }
\end{array}\right. \\
& =\left\{\begin{array}{l}
\tilde{h}[n]+\tilde{w}[n], \quad n \in\{0,1, \cdots, N t-1\} . \\
0, \quad \text { remaining }
\end{array}\right.
\end{aligned}
$$




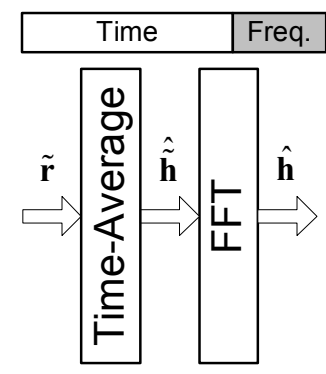

Fig. 2. Direct TD pilot-aided channel estimation.

The vector $\tilde{\mathbf{h}}_{\mathbf{L S}}$, whose elements are defined by (21), is made-up of $N f$ scaled replicas of the CIR contaminated by noise. Moreover, the vector elements defined by (21) and (22) do not depend on the data component. As expected, the data component present in $\tilde{\mathbf{r}}$ was eliminated by the TD equivalent LS estimate processing.

Rewriting (18) in the form

$$
\begin{aligned}
\hat{\tilde{h}}_{L S}[n] & =\tilde{p}[n] * \tilde{r}[n] \\
= & \frac{1}{N f} \sum_{m=0}^{N f-1}\left(\begin{array}{l}
\sum_{l=-\infty}^{+\infty} \tilde{h}[l] \tilde{d}[n-m N t-l] \\
+\frac{1}{N f} \delta[n-m N t] * \sum_{l=0}^{N f-1} \tilde{h}[n-l N t] \\
+\tilde{w}[n-m N t]
\end{array}\right),
\end{aligned}
$$

and replacing in (16),

$$
\begin{aligned}
\hat{\tilde{h}}[n] & =\left\{\begin{array}{l}
\sum_{m=0}^{N f-1}\left(\begin{array}{l}
\sum_{l=-\infty}^{+\infty} \tilde{h}[l] \tilde{d}[n-m N t-l] \\
+\frac{\sum_{l=0}^{N f-1} \tilde{h}[n-l N t-m N t]}{N f} \\
+\tilde{w}[n-m N t]
\end{array}\right), n \in\{0,1, \cdots, N t-1\} \\
0, \quad \text { remaining }
\end{array}\right) \\
& =\left\{\begin{array}{l}
\sum_{m=0}^{N f-1}(\tilde{r}[n-m N t]), n \in\{0,1, \cdots, N t-1\} \\
0, \quad \text { remaining }
\end{array}\right.
\end{aligned}
$$

yields an important result. All the processing required by the method in [4] can be reduced to a sum operation directly in TD and a DFT. The new method is depicted in Fig. 2.

Most DFT-based interpolation methods [2], [6], [7] can be directly applied at no extra processing cost.

In terms of complexity, the proposed method does not use multiplications and requires a total of $N t N f=N c$ complex addition, resulting in only 1 complex addition per tap to get the CIR estimate. The complexity of the method in [4] is summarized in Table I. To calculate the CIR it requires a total of $2+\frac{\log _{2}(N c)}{2}$ complex multiplications and $\log _{2}(N c)$ complex addition per tap. The proposed method represents a considerable improvement in terms of complexity.

TABLE I. LS-DFT CHANNEL ESTIMATION COMPLEXITY (OPERATIONS PER TAP).

\begin{tabular}{|c|c|c|c|c|}
\cline { 2 - 5 } \multicolumn{1}{c|}{} & $\begin{array}{c}\text { FD Zero } \\
\text { Forcing }\end{array}$ & IDFT [8] & $\begin{array}{c}\text { TD low-pass } \\
\text { window }\end{array}$ & $\begin{array}{c}\text { Required operations } \\
\text { per tap }\end{array}$ \\
\hline $\begin{array}{c}\text { Complex } \\
\text { multiplication }\end{array}$ & 1 & $\frac{\log _{2}(N c)}{2}$ & 1 & $2+\frac{\log _{2}(N c)}{2}$ \\
\hline $\begin{array}{c}\text { Complex } \\
\text { addition }\end{array}$ & - & $\log _{2}(N c)$ & - & $\log _{2}(N c)$ \\
\hline
\end{tabular}

\section{Simulation RESUlts}

A simulation scenario was implemented where $N c=1024$ subcarriers were QPSK modulated. The adopted frame has a pilot separation $N f=4$ and the system's sampling time $\Delta t=25 n s$.

This work focuses on the channel estimation of the received symbols carrying both pilots and data and does not consider the problem of estimating the channel when data symbols (with no pilots) are transmitted.

Fig. 3a shows the vectors $\tilde{\mathbf{r}}$ and $\hat{\tilde{\mathbf{h}}}$, when using a BRAN-A channel model [9] with the receiver's signal to noise ratio set to $S N R=6 d B$. It is intended to show that the proposed method eliminates the data component and attains the CIR estimate with the operation in (24). This is further enhanced by Fig. 3b, which shows that the previous vector $\hat{\tilde{\mathbf{h}}}$ and the received vector $\tilde{\mathbf{r}}_{\mathbf{p}}$, resulting from the transmission of $\tilde{\mathbf{s}}$ in (7) with the data vector $\tilde{\mathbf{d}}$ set to zero, under the same transmission conditions, perfectly overlap.

To validate the proposed method, channel estimation
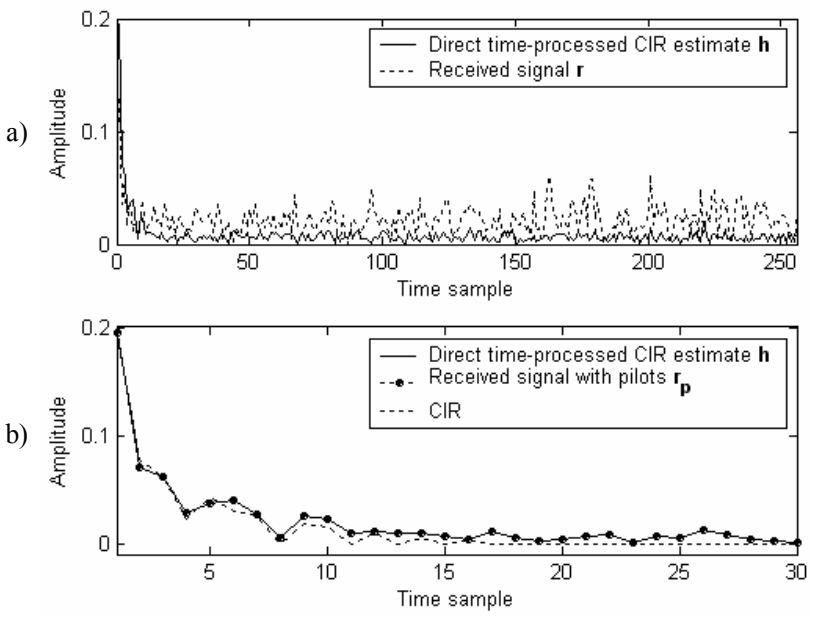

Fig. 3. Time-domain received vectors. 


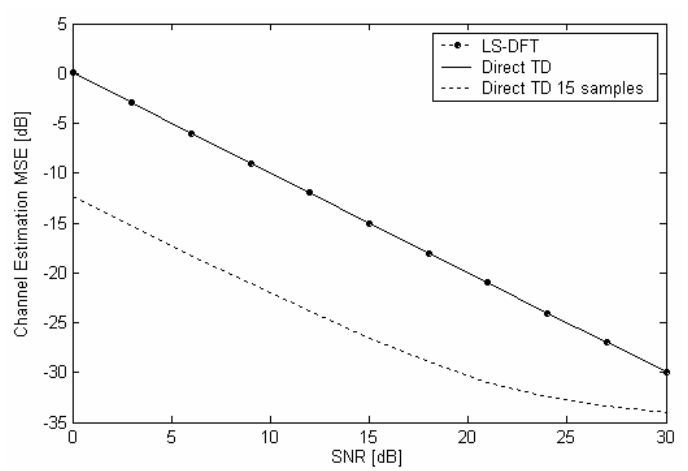

Fig. 4. Channel estimation MSE performance evaluation.

simulations were performed for SNRs in the range of $0 \mathrm{~dB}$ to $30 \mathrm{~dB}$, and compared with the method in [4]. The results are depicted in Fig. 4, identified as "LS-DFT" and "Direct TD". The channel estimation MSE for both methods is equivalent for all SNR values, confirming once again (24).

As previously referred, the proposed method can incorporate other published techniques to achieve better performance. To exemplify such a combination, the curve identified as "Direct TD 15 samples" in Fig. 4 is the result of keeping the 15 most significant taps of the CIR using the scheme in [3]. The significant improvement was achieved at no extra processing cost.

\section{CONCLUSIONS}

We have presented a method to estimate the channel's frequency response using the received data in TD with a significant reduction of the required processing. The method is able to extract the data component by applying simple linear processing on the received vector.

Furthermore this method can incorporate other published schemes, at no added complexity, to improve the estimate of the channel.

\section{REFERENCES}

[1] R. V. Nee, R. Prasad, OFDM for Wireless Multimedia Communications, $1^{\text {st }}$ edition, Artech House, 2000.

[2] C. S. Yeh, Y. Lin, "Channel estimation using pilots tones in OFDM systems," IEEE Transactions on Broadcasting, vol. 45, pp. 400-409, December 1999.

[3] H. Minn, V. Bhargava, "An investigation into time-domain approach for OFDM channel estimation," IEEE Transactions on Broadcasting, vol. 46, no. 4, pp. 240-148, December 2000.

[4] A. Chini, M. S. El-Tanany, S. A. Mahmoud, "Transmission of high rate ATM packets over indoor radio channels," IEEE Journal on Selected Areas in Communications, vol. 14, nº4, April 1996.

[5] P. Hoeher, S. Kaiser, P. Robertson, "Two-dimensional pilot-symbolaided channel estimation by Wiener filtering," Proc. ICASSP97, Munich, Germany, pp. 1845-1848, April 1997.

[6] A. Chini, "Multicarrier modulation in frequency selective fading channels," Ph.D. dissertation, Carleton University, Canada, 1994.

[7] O. Edfors, "Low-complexity algorithms in digital receivers," $\mathrm{Ph} . \mathrm{D}$. dissertation, Lulea University of Technology, Sweden, 1996.

[8] A. Oppenheim, R. Schafer, Discrete-time Signal Processing, $2^{\text {nd }}$ edition, Prentice Hall, 1999.

[9] ETSI Project Broadband Radio Access Networks (BRAN), HIPERLAN Type 2, Technical specification; Physical layer, October 1999. 